Central Washington University

ScholarWorks@CWU

$5-2-2008$

\title{
Automated Vector-to-Raster Image Registration
}

Boris Kovalerchuk

Peter Doucette

Gamal Seedahmed

Robert Brigantic

Michael Kovalerchuk

See next page for additional authors

Follow this and additional works at: https://digitalcommons.cwu.edu/compsci

Part of the Computer Sciences Commons, and the Geographic Information Sciences Commons 
Authors

Boris Kovalerchuk, Peter Doucette, Gamal Seedahmed, Robert Brigantic, Michael Kovalerchuk, and Brian Graff 


\title{
Automated Vector-to-Raster Image Registration
}

\author{
Boris Kovalerchuk $^{\mathrm{ab} *}$, Peter Doucette ${ }^{\mathrm{c}}$, Gamal Seedahmed ${ }^{\mathrm{d}}$, \\ Robert Brigantic $^{\mathrm{e}}$, Michael Kovalerchuk ${ }^{\mathrm{b}}$, Brian Graff ${ }^{\mathrm{f}}$ \\ ${ }^{a}$ Central Washington University, Ellensburg, WA 98926-7520 \\ ${ }^{\mathbf{b}}$ BKF Systems, 8241 S. 123 St., Seattle, WA, 98178, \\ ${ }^{\mathrm{c}}$ ITT Advanced Engineering \& Sciences, 2560, Huntington Ave, Alexandria, VA 22303, \\ ${ }^{\mathrm{d}} \mathrm{NG} 4,10780$ N. Preserve Way 308, Miramar, FL, 33025, \\ ${ }^{\mathrm{e}}$ Battelle Pacific Northwest Division, Richland, WA 99352, \\ ${ }^{f}$ US Army Topographic Engineering Center, Fort Belvoir, VA
}

\begin{abstract}
The variability of panchromatic and multispectral images, vector data (maps) and DEM models is growing. Accordingly, the requests and challenges are growing to correlate, match, co-register, and fuse them. Data to be integrated may have inaccurate and contradictory geo-references or not have them at all. Alignment of vector (feature) and raster (image) geospatial data is a difficult and time-consuming process when transformational relationships between the two are nonlinear. The robust solutions and commercial software products that address current challenges do not yet exist. In the proposed approach for Vector-to-Raster Registration (VRR) the candidate features are auto-extracted from imagery, vectorized, and compared against existing vector layer(s) to be registered. Given that available automated feature extraction (AFE) methods quite often produce false features and miss some features, we use additional information to improve AFE. This information is the existing vector data, but the vector data are not perfect as well. To deal with this problem the VRR process uses an algebraic structural algorithm (ASA), similarity transformation of local features algorithm (STLF), and a multi-loop process that repeats (AFE-VRR) process several times. The experiments show that it was successful in registering road vectors to commercial panchromatic and multi-spectral imagery.
\end{abstract}

Keywords: image, vector data, registration, conflation, alignment

\section{INTRODUCTION}

Alignment of vector (feature) and raster (image) geospatial data is a difficult and time-consuming process when transformational relationships between the two are nonlinear. Figure 1 illustrates misalignment problems that are nonlinear in general, i.e., may require adjustments of individual components versus global systematic adjustments. To further complicate the problem, candidate data for registration may have inaccurate and contradictory geo-references or not have them at all. Different and unknown rotations, disproportional scales, uncontrolled noise, different modalities, are all fundamental challenges for robust registration.

There are certain applications that require a high degree of accuracy when integrating (fusing) multiple data layers. In figure 1a, the degree of misalignment between road vectors with imagery varies locally within a range of 0-30 meters. In this case, the source of the misalignment is caused by the original extraction of the road vector layer being based upon outdated imagery. Therefore, realignment of the road vector layer is needed with respect to the more recently acquired image, which is presumed more geospatially accurate than its predecessor image. In this context, it is particularly noteworthy that many vector-to-raster inconsistencies exist at popular data websites such as Google ${ }^{\mathrm{TM}}$ Maps, Yahoo! ${ }^{\circledR}$ Maps, MapQuest ${ }^{\circledR}$, and Microsoft Virtual Earth ${ }^{\mathrm{TM}}$ (see Figure 1b). The major reason of misalignment is the

*bkf1@BKFSystems.com; phone/fax 509 857-2500;

Algorithms and Technologies for Multispectral, Hyperspectral, and Ultraspectral Imagery XIV, edited by Sylvia S. Shen, Paul E. Lewis, Proc. of SPIE Vol. 6966, 69660W, (2008) · 0277-786X/08/\$18 · doi: 10.1117/12.778431 
oversimplified method of co registration of vector an raster data shown in Figure 2 that uses only few corner points and ignores intermediate points.

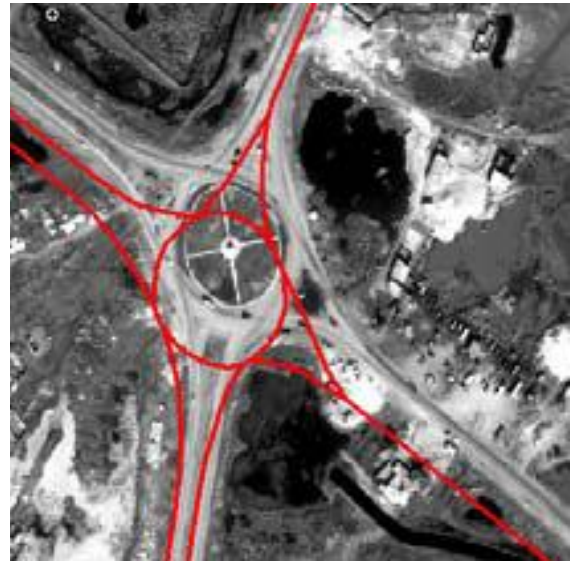

(a)

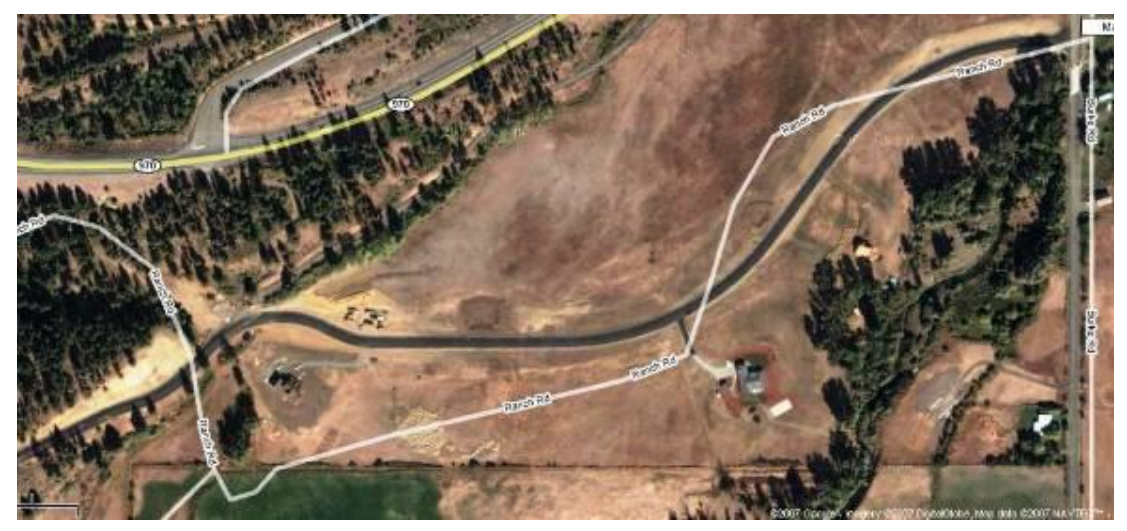

(b)

Fig. 1. (a) Examples of nonlinear misalignment between UVMap road vectors (red), and commercial imagery. (images CDigitalGlobe, 2006), (b) Image-map overlay in hybrid Google maps with several visible misalignments.
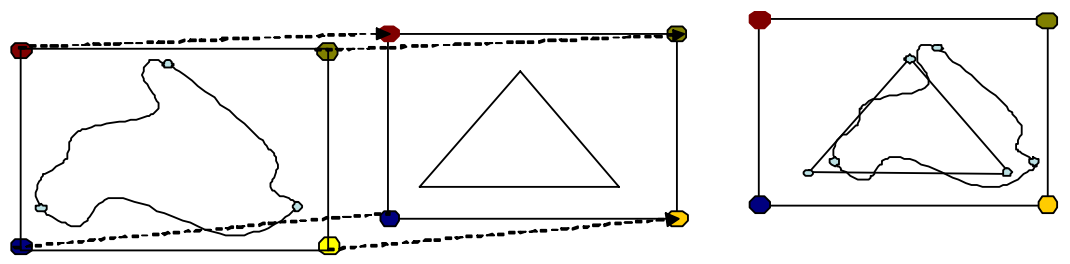

(a)
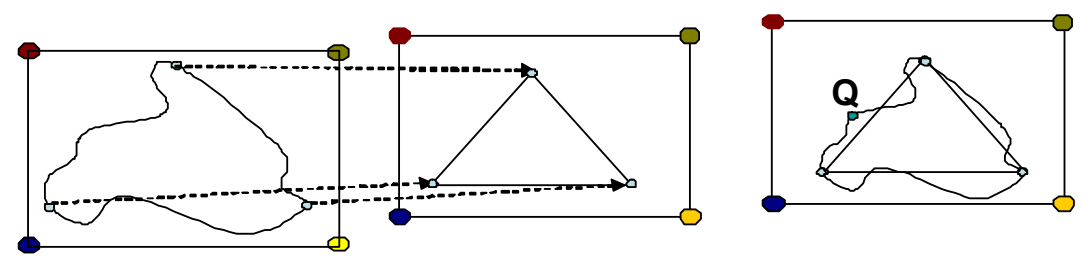

(b)

Fig. 2. Control point based fusion of imagery and maps: (a) image corner points, (b) feature-based points.

In both cases (a) and (b) when only intersection points are matched, the match of the intermediate point $\mathrm{Q}$ is not known for such highly non-linear match situation.

Vector and raster data registration and conflation problems are discussed extensively in the literature, e.g., ${ }^{1-3 .}$ The sequential steps of feature extraction, feature matching, parameters estimation, and resampling have evolved into a common paradigm for automated image matching and registration ${ }^{4}$. In general, automated vector-to-image registration methods are not nearly as robust compared to raster-to-raster registration as shown $\mathrm{in}^{5}$, or vector-to-vector conflation methods.

The problem domain addressed in this paper is characterized as follows:

- Register Urban Vector Map (UVMap) data to panchromatic (1m) and/or multispectral (MSI) (4m) commercial satellite imagery.

- Focus on road vector layers.

- Extent of misalignment is in the range of $0-30 \mathrm{~m}$, and transformation is nonlinear. 
- Misalignment needs to be corrected for at road intersections (nodes), and at intermediate points between road intersections (shape points).

In our approach for Vector-to-Raster Registration (VRR) the candidate features are auto-extracted and vectorized from imagery, which are the basis to compare against existing vector layer(s) to be registered. Given that available automated feature extraction (AFE) methods quite often produce false features and miss some features, we exploits additional information available in the vector-to-raster registration task. This information is the vector data, but the vector data are not perfect as well. The VRR process uses an algebraic structural algorithm (ASA) and similarity transformation of local features algorithm (STLF). This paper demonstrates is able to register road vectors to commercial panchromatic and multi-spectral imagery.

The paper is organized as follows. In section 2 at first, we discuss limits of robust and reliable VRR and requirements for this. Section 3 presents the general technical approach framework. Sections 4 presents automated road extraction and performance evaluation. Section 5 describes experiments with VRR algorithms.

\section{LIMITS OF ROBUST AND RELIABLE VRR}

The fundamental question is how critical is the quality of feature extraction for VRC systems success. The opinions range from it is vital to the almost opposite opinion that any reasonable but noisy AFE is sufficient for VRC. Below we clarify this issue.

Many automated and semi-automated approaches exist. The accuracies of change detections by pixels learning with Feature Analyst (FA) method are reported in $^{11}$ : Aircraft $37.4 \%$, buildings $35.8 \%$, vehicles $28.1 \%$, (all less then $50 \%$ ). This paper has no direct data for roads but our experiments show that it is in the same range. The semi-automatic learning FA method requires minor intervention for selection of training pixels. That is, one might be willing to spend some time pulling some pixels (the easy ones) from an image to be used for training purposes if this leads to a greatly increased overall accuracy of the extraction compared to a fully automated method. The semi-automatic method proposed by GeoEye requires more user guidance that FA but can be accurate. The level of robustness of current fully automated road extraction tools typically is lower. Therefore, while fully automated road extraction is a worthy goal, the semi-automated approaches increase feature extraction accuracy right away. Some scenes lend themselves toward total automation more than others. The semi-automated approaches must meet the requirement to be as minimal and as simple as possible for the user. The user should provide minimal but sufficient number of roads to match to the road vector datasets. Testing and comparing automatic and semi-automatic methods requires a side-by-side comparison of results in terms of accuracy, time and other requirements to be able to judge was it worth the added time.

The practical goal is to avoid a complete extraction of the road network. The expectation/hypothesis is that a reasonable percentage of inliers (say 30\%) can be good enough to solve the conflation problem with minimal semi-automated solution involved. Our experiments show that data can be conflated/registered having much less than $30 \%$ of roads. However, a user should be aware that it was done with, say mentioned $30 \%$ of roads extracted and for the $70 \%$ or roads the mach was not justified yet. The user should examine that $70 \%$ of roads and be ready to spend time. Only after the user confirms that the result is "good enough" for his/her purposes it can be used. The user may decide to examine only $1 \%$ of randomly selected roads to say that this is "good enough," but it is user's risk. Alternatively, an automated system can try to measure "a reasonable percentage" and "good enough". This approach leads to the concept of the "measure of confidence." If such measure is low then it is not "good enough" and a user should examine almost every road (and spend a lot of time). The low measure of confidence may require examining conflation result at almost every road. In contrast having majority of roads with a high level of confidence a user needs to examine just few outlier roads saving users time.

The "good enough" measure is not universal but task-specific. The number or percentage of roads extracted is the quantitative factor, and that might be high for an analyst whose job it is to accurately locate and map the transportation network for a large area. On the other hand, there are the "right" roads to extract for an analyst who is focused on a specific local target - hence this is more of a qualitative or "good enough" aspect of the problem. Such user needs only a very small set of roads extracted with great accuracy. While the solution approach is task-specific, it does not deny the 
need to have the full capability to do a large area road network. If we have a perfect AFE we do not need VRR. Can we reach the perfect AFE using only available images? This is quite unrealistic expectation at this moment. Assume that a weak AFE allows high quality or perfect VRR. How can we ensure this for large dataset, not only for a small image that a person can examine in depth? The question is: "What is the limit of weak AFE?" In other words, "How weak AFE can be to allow high-quality or perfect VRC?"

Let us start from the extreme case where AFE did not extract any road correctly, that is none of the pixels match, and the distance to the matching pixels is greater than a reasonable for the application threshold. It is hard to expect that this extreme AFE can be useful for VRC.

Now we will start a more detailed analysis of the situation by introducing a notation:

- $\mathrm{x}$ is the number of road pixels extracted correctly,

- $\mathrm{z}$ is the number of roads pixels missed in the extraction process

- $y$ is the number of false positive extracted pixels (non-road pixels recognized as a road pixel).

- $\mathrm{N}$ is the correct number of road pixels

Let us consider the following assumptions:

(A1) $\mathrm{y}=0$ (no false positive pixels points in vector data that is every point in vector data has a matching point in raster image).

(A2) The class of transforms $\left\{\mathrm{F}_{\mathrm{p}}\right\}$ from vector to raster is selected correctly

(A3) The number $\mathrm{x}$ of the extracted points is sufficient to identify specific values of parameters $\mathrm{p}$ of the transform Fp.

Statement 1. If A1-A3 are satisfied then VRR function $\mathrm{F}_{\mathrm{p}}$ provides a correct transformation of vector data to raster image.

Statement 2. If $\mathrm{x} / \mathrm{N}=1$ (all road pixels are extracted) and $\mathrm{y}=0$ (perfect $\mathrm{AFE}$ ) and conditions A1-A3 are satisfied then VRR function $F_{p}$ provides a correct transformation of Vector to Raster, but $F_{p}$ is not needed due to perfect feature extraction.

Statement 3. If $\mathrm{x}=0$ (AFE fails) then parameters of VRR function $\mathrm{F}_{\mathrm{p}}$ cannot be identified.

Statement 1 covers interesting situation when $0<\mathrm{x} / \mathrm{N}<1$ and statements 2 and 3 cover two trivial extremes. Now we want to analyze assumption A1-A3 to check how realistic they are and how they can be tested.

How can we know that A1 holds? It can be a judgment based previous experiments with the same type of data (e.g. for $\mathrm{x} / \mathrm{N} \approx 0.4$ as reported $\mathrm{in}^{11}$ ). A time consuming option is a direct examination of AFE results by the user. To test A2 an experiment on the small patch of data can be conducted or results of previous experiments can be used. For A3 this can be derived from the properties of the class $\{\mathrm{Fp}\}$ such as affine transforms.

Now we need to consider a situation when some of A1-A3 failed in the described above tests. If $y>0$ then we need a tool to filter these pixels out, e.g., some roof pixels were recognized as road pixels. It can be done if A2 holds using vector data as a guide. If both $\mathrm{A} 1$ and $\mathrm{A} 3$ fail then external tools are needed to filter out y pixels. These considerations show that for robust solution of VRR problem in a complex situation external knowledge is needed that may lead to semi-automated system. In essence, the analysis above outlines the area where a fully automated method can succeed. If A1-A3 test fail then interactive semi-automatic solutions would be preferred to ensure the robust VRR result.

\section{GENERAL TECHNICAL APPROACH}

Below we outline three stepwise categories of our VRR technology: preprocessing, processing, and post-processing steps (see Figure 3). The goal of preprocessing is to identify and assess metadata and knowledge that are available but may not be contained in the imagery or vector data explicitly. Such information if available can guide processing steps 
that actually conflate/align data. The guidance serves several important functions such as mimicking a successful human pre-conflation process. Humans always have some meta-knowledge before starting conflation. Preprocessing can also shorten conflation time significantly; for example, if we know that unknown rotation is less than 30 degrees, then the search space will be smaller than the full search space. The preprocessing steps often are skipped with naïve hope that a processing conflation algorithm will do magic without any background knowledge. Anther advantage of explicit introduction of a preprocessing step is enhancing problem understanding. This may or may not improve processing results immediately, but it may explain why processing results are not good and magic did not happen. This can guide development of new or better algorithms, making the whole process of improvement consistent and continuous. To be able to do this, we want to build a software system with additional capabilities to support failure recording and analysis based on preprocessing information. Preprocessing steps are designed to be interactive sessions; e.g., a user may be asked to answer multiple-choice questions. Processing steps are designed to integrate two well-developed methodologies for conflation: algebraic structural algorithms (ASA) and similarity transformation of local features (STLF). Both methodologies match and transform imagery and vector data. The post-processing steps are for approving/disapproving and fixing match and alignment by the user.

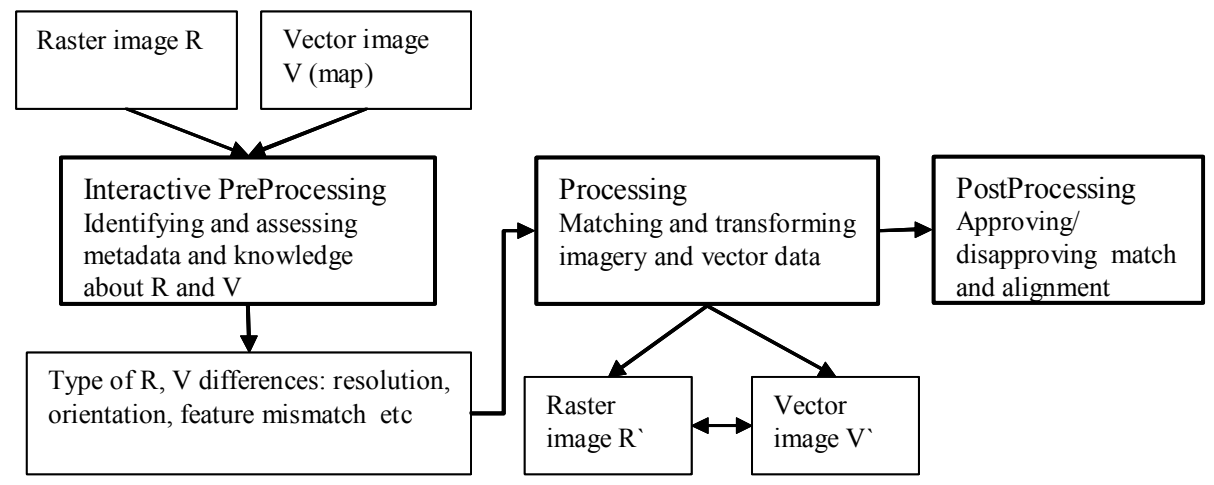

Fig. 3. General technical approach framework

To test the technology we use ground truth roads that are extracted manually and/or semi-automatically from imagery and then manually corrected and verified. These data allow us to evaluate the quality of (a) automated feature (road) extraction algorithms, (b) road representation in the in the vector data, (c) road representation in the imagery (some roads can be obscured by vegetation, flood, clouds, etc), and (d) automated vector to rater conflation algorithms. Finally, ground truth data are the base for building an objective measure of the confidence of raster-vector conflation. Two roads may not match due to: (1) actual difference of roads, (2) weaknesses of the algorithms (including feature extraction algorithm), (3) errors in source vector data, (4) problems with raster data (low resolution, occlusion etc).

How can AFE and VRC can assist each other? AFE assists VRC in a natural way, AFE results are the base of the vector to vector to conflation. Simply without AFE VRC is impossible. The question is: "How it can be done in the opposite direction?" When VRC is produced then we can examine roads extracted by AFE and evaluate how close they are and how similar they are to vector roads after roads have been moved to new location. The advantage of such examination is that we obtain some additional information on where the road can be. This information is independent of the raster itself and can guide a deep analysis of specific pixels in a specific image region. For instance, we can use multispectral imagery to analyze very specific pixels where the road can be obscured by vegetation. This process can run as a loop of AFE and VRC.

\section{AUTOMATED ROAD EXTRACTION AND PERFORMANCE EVALUATION}

Our approach to road extraction is to consider and assess several automated methods in the literature and marketplace. The goal is to maximize the level of automation, while concurrently optimizing the confidence level of the output. Our hypothesis is that using only higher confidence output from road extraction algorithms will reduce false positive rate of extraction, and subsequently avoid confusion for registration of an existing misaligned road layer. The tradeoff is a 
higher false negative rate of extraction. Thus, a key research question is determining what extraction rates of completeness and correctness are optimal for registration. Sensitivity analysis of road extraction parameters can provide insight into this issue.

In this section we experiment with the Anti-parallel Centerline Extraction (ACE) road extraction algorithm ${ }^{6}$. In context of VRR, the objective of ACE is to provide road centerline "seed" segments, which are defined as high confidence road segments according to predefined shape and topology criteria. Sensitivity analysis of ACE parameters was performed via design of experiments (DOE).

\subsection{Design of Experiments}

To help determine optimal and/or robust parameter (or factor) settings in applying the ACE algorithm, a Design of Experiments (DOE) approach was considered. DOE is a systematic approach to explore response surfaces which are a function of controllable and uncontrollable input factors. In the case of the ACE algorithm, all of the input factors are parameter settings which are controllable as specified by the user. The systematic methodology behind DOE allows one to explore this surface and look for parameter settings that yield desirable output results in an efficient manner. Using the DOE methodology as described in various sources ${ }^{13-14}$, we established an initial screening design for our ACE parameters of interest and then determined suitable low and high values for each of these as shown in Table 1. The reader should refer to $^{6-7}$ for a more complete description of these ACE parameters and corresponding test images.

Fig. 4 shows a graphical plot of main effects for each of the 10 ACE parameters under consideration. This plot provides a visual clue as to the relative significance of parameters in that each line represents the average response when a particular parameter is at its low setting and high setting. The more a line departs from a horizontal position (meaning there is no difference between the parameter being at its low or high setting) the more significant the effect. As seen, the three most significant effects for this scene are C: sigma (standard deviation of Gaussian smoothing filter applied before Canny filter), $\mathrm{H}$ : eccentricity threshold, and E: maximum feature width respectively.

Table 1. DOE Factors and Uncoded Values for 10 Factor Fractional Factorial Design

\begin{tabular}{cclcc}
\hline \hline $\begin{array}{c}\text { Factor } \\
\text { Symbol }\end{array}$ & $\begin{array}{c}\text { Factor } \\
\text { Label }\end{array}$ & \multicolumn{1}{c}{ Shorthand Factor Name } & $\begin{array}{c}\text { Low } \\
\text { Value }\end{array}$ & $\begin{array}{c}\text { High } \\
\text { Value }\end{array}$ \\
\hline$T_{L}$ & $A$ & low threshold & 0.08 & 0.14 \\
$T_{H}$ & $B$ & high threshold & 0.20 & 0.40 \\
$\sigma$ & $C$ & sigma & 1.00 & 6.00 \\
$w_{\min }$ & $D$ & min feature width (m) & 2 & 8 \\
$w_{\max }$ & $E$ & max feature width (m) & 10 & 16 \\
$\alpha$ & $F$ & alpha (deg) & 20.0 & 60.0 \\
$c c_{\min }$ & $G$ & min number connected pixels & 2 & 12 \\
$e c c T h r e s h$ & $H$ & eccentricity threshold & 0.70 & 0.90 \\
$g$ & $J$ & gradient orientation angle & 1 & 2 \\
$p$ & $K$ & Perpendicularity & 0 & 1 \\
\hline \hline
\end{tabular}

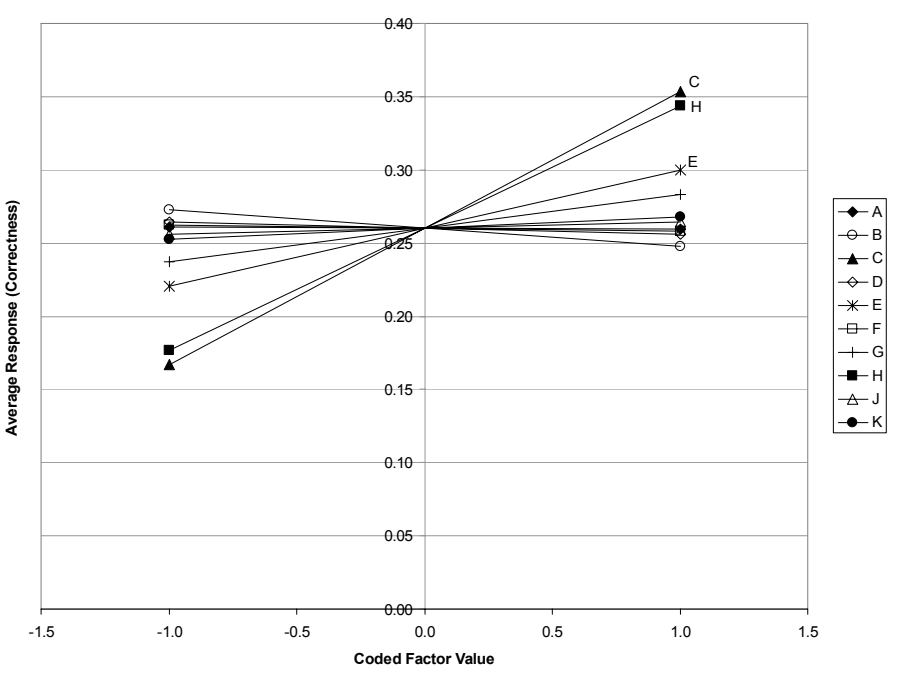

Fig. 4. Main effects plot of ACE input parameters

In summary, DOE revealed that the most statistically significant variables influencing the accuracy of road extraction of the sampled images were sigma (Gaussian smoothing parameter), eccentricity threshold, and the minimum number of connected pixels. However, the specification of a suitable constrained range of parameter values over different scenes was problematic. In general, the most important metric to favor is correctness since the results of ACE will be used in as training samples for the k-means clustering algorithm. Therefore, parameters should be chosen such that correctness is maximized. It was found that despite having a relatively low number of pixels identified by ACE, if the correctness was high, the clustering method was able adequately identify roads versus non-roads from the input image. 


\subsection{Road Extraction Experiments with ACE}

Figure 5 demonstrates road extraction results from application of ACE. Pane (a) shows a section of Quickbird panchromatic image, with manually extracted road centerline vectors overlaid in red. The dotted yellow vectors demonstrate the misalignment of the existing vector data. In pane (b), a $5 \mathrm{~m}$ buffer has been extended around the centerline vectors from pane (a) to provide an evaluation buffer. Output from ACE is shown in red in pane (b). The goal of ACE in this experiment is to provide road centerline "seed" segments, which are defined as high confidence road segments according to predefined shape and topology criteria. Pane (c) shows smoothed ACE output in red. The smoothing algorithm is a standard generalization technique for vector data. Smoothing is needed to calculate the linear length of extracted output that is consistent with the length calculation of manually extracted "truth" for comparative evaluation. Since ACE is a pixel-based extraction method, its raw output will manifest pixilated zigzag artifacts as shown in pane (b). Such artifacts artificially increase the linear length calculations. Pane (d) shows the ACE output separated in to true positive segments (blue), and false positive segments (dotted red), based upon the $5 \mathrm{~m}$ evaluation buffer. The size of the buffer is determined empirically to allow for some tolerance between manual and algorithm-based extraction. In general, the size of the evaluation buffer is directly proportional to the true positive rate of extraction, and inversely proportional to the false positive rate.

Empirical performance metrics are based upon standard calculations performed in the literature ${ }^{6,12}$, which can be done in raster or vector space. For example, the performance of the ACE output in pane (d) is calculated as:

$$
\begin{aligned}
& \text { completeness }=\mathrm{TP}_{\mathrm{L}} / \mathrm{GT}_{\mathrm{L}}=1330 \mathrm{~m} / 9133 \mathrm{~m}=15 \% \\
& \text { correctness }=\mathrm{TP}_{\mathrm{L}} /\left(\mathrm{TP}_{\mathrm{L}}+\mathrm{FP}_{\mathrm{L}}\right)=1330 \mathrm{~m} /(1330 \mathrm{~m}+506 \mathrm{~m})=72 \%
\end{aligned}
$$

$\mathrm{TP}_{\mathrm{L}}, \mathrm{FP}_{\mathrm{L}}, \mathrm{GT}_{\mathrm{L}}$, are defined as true positive length, false positive length, and ground truth length respectively. These performance metrics can serve as the basis for sensitivity analysis to determine how to optimally tune the road extraction algorithm to support vector-to-vector registration. For this particular ACE parameter set, the completeness is relatively low at $15 \%$, but correctness is relatively high at $72 \%$.

\section{EXPERIMENTS WITH VECTOR TO RASTER REGISTRATION ALGORITHMS}

Experiments were performed with two VRR methods: 1) Algebraic Structural Analysis (ASA) algorithm, and 2) Similarity Transformation of Local Features (STLF). Tests with several chipped regions from panchromatic and multispectral imagery (MSI), with corresponding UVMap vector data. Test images were rescaled from 11bit, to 8bit for operational efficiency. Several image enhancement methods were considered, including pan sharpening, and principal component analysis.

\subsection{Algebraic Structural Analysis (ASA) algorithm}

The algebraic Structural Analysis (ASA) algorithms ${ }^{3,7-8}$ are proposed to conflate (i) linear features that are modeled as open contours (algorithm ASA and (ii) region features (that are modeled by closed contours and areas (algorithm ARC). Roads can be modeled by both open and closed contours depending on particular data. These algorithms can work together with linear feature extraction tools for (i) and segmentation tools for (ii). Both ASA and ARC algorithms are parts of a general Algebraic Framework. Advantages of algebraic approach relative to pure geometric and topological approach for feature matching are presented in $^{3}$. 


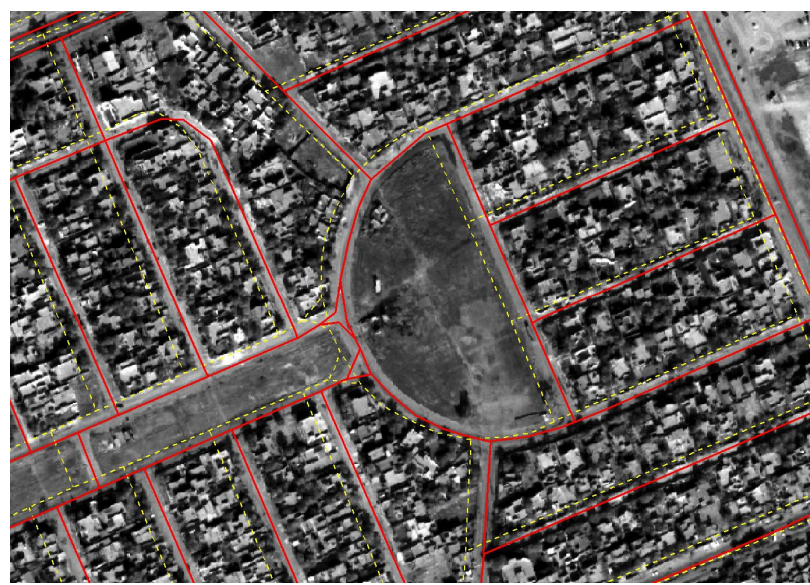

(a)

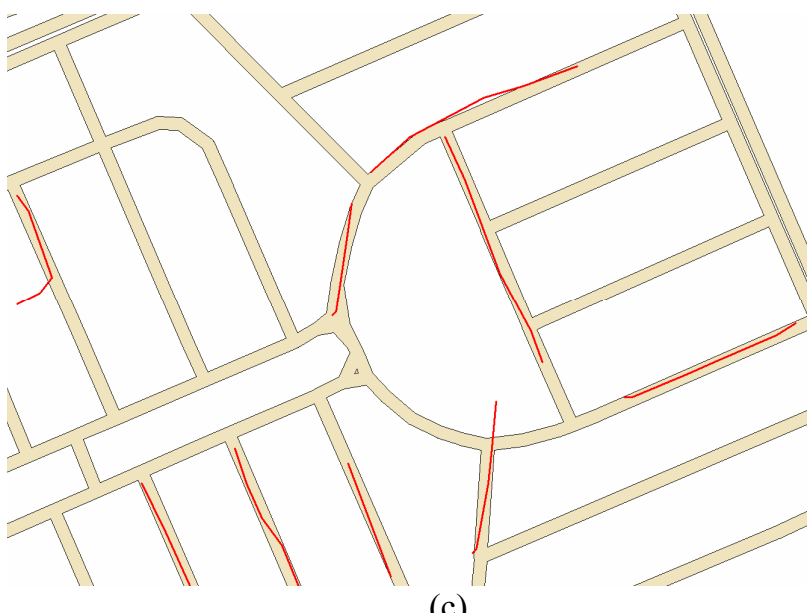

(c)

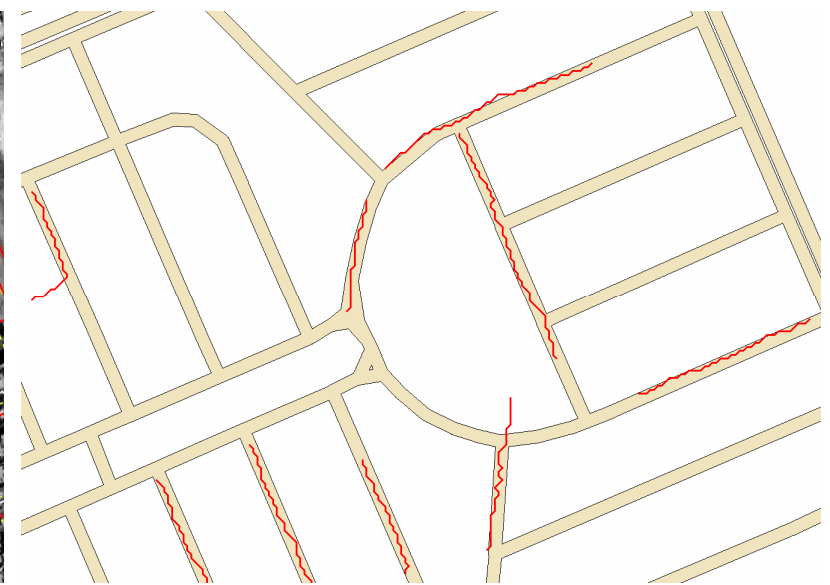

(b)

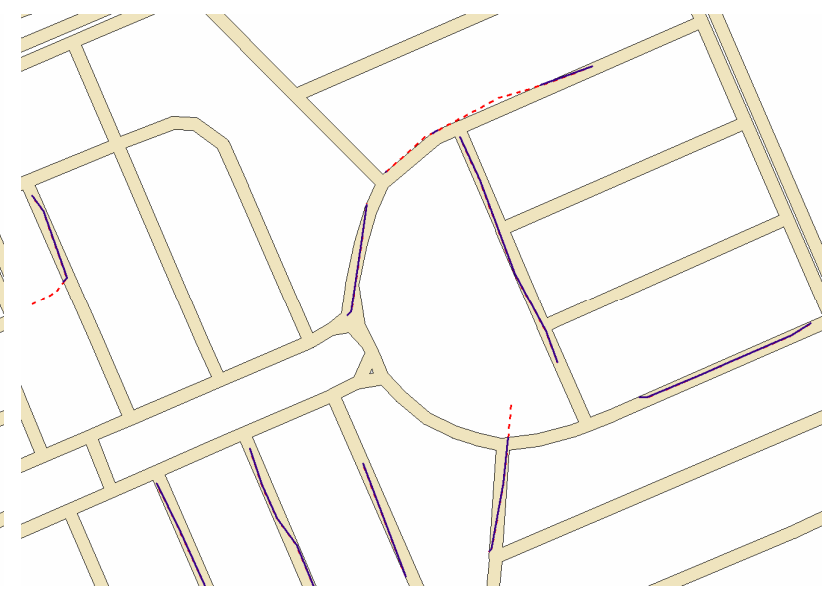

(d)

Fig. 5. Automated road extraction output and evaluation. (a) Quickbird panchromatic image (@ DigitalGlobe) with new manually extracted road centerlines (red), and older and misaligned road vectors (dotted yellow); (b) manually extracted centerlines buffered by $5 \mathrm{~m}$ (tan), with high confidence ACE centerline seed segments (red); (c) smoothed ACE output (red) for more consistent evaluation; (d) ACE seed output separated into true positive vectors (blue) and false positive vectors (dotted red).

The algorithm used for linear features is the binary sequential division (BSD) algorithm. BSD assumes that input data contain at least one well-defined linear feature that can be presented as a polyline (continuous chains of linear intervals). A feature on one image might be only a portion of the same feature on another image. Also features might overlap or have no match at all. It is further assumed that these well-defined linear features can be relatively easy extracted. For practical reasons, we would prefer images with five well-defined linear features that can be presented as polylines. This provides more robust and confident conflation. Next versions of the algorithm will work with polylines that have gaps.

Fig. 6 shows results of VRR produced by ASA. Pane (a) shows the input QuickBird MSI scene. Pane (b) shows the original road vectors (yellow) superimposed with semi-automated feature extraction output by Feature Analyst ${ }^{\circledR}$ (white). Pane (c) shows the road extraction output from Feature Analyst. Pane (d) shows the final adjustment for original vectors (green), superimposed with Feature Analyst vectors (cyan), and ASA matched segments (black). 

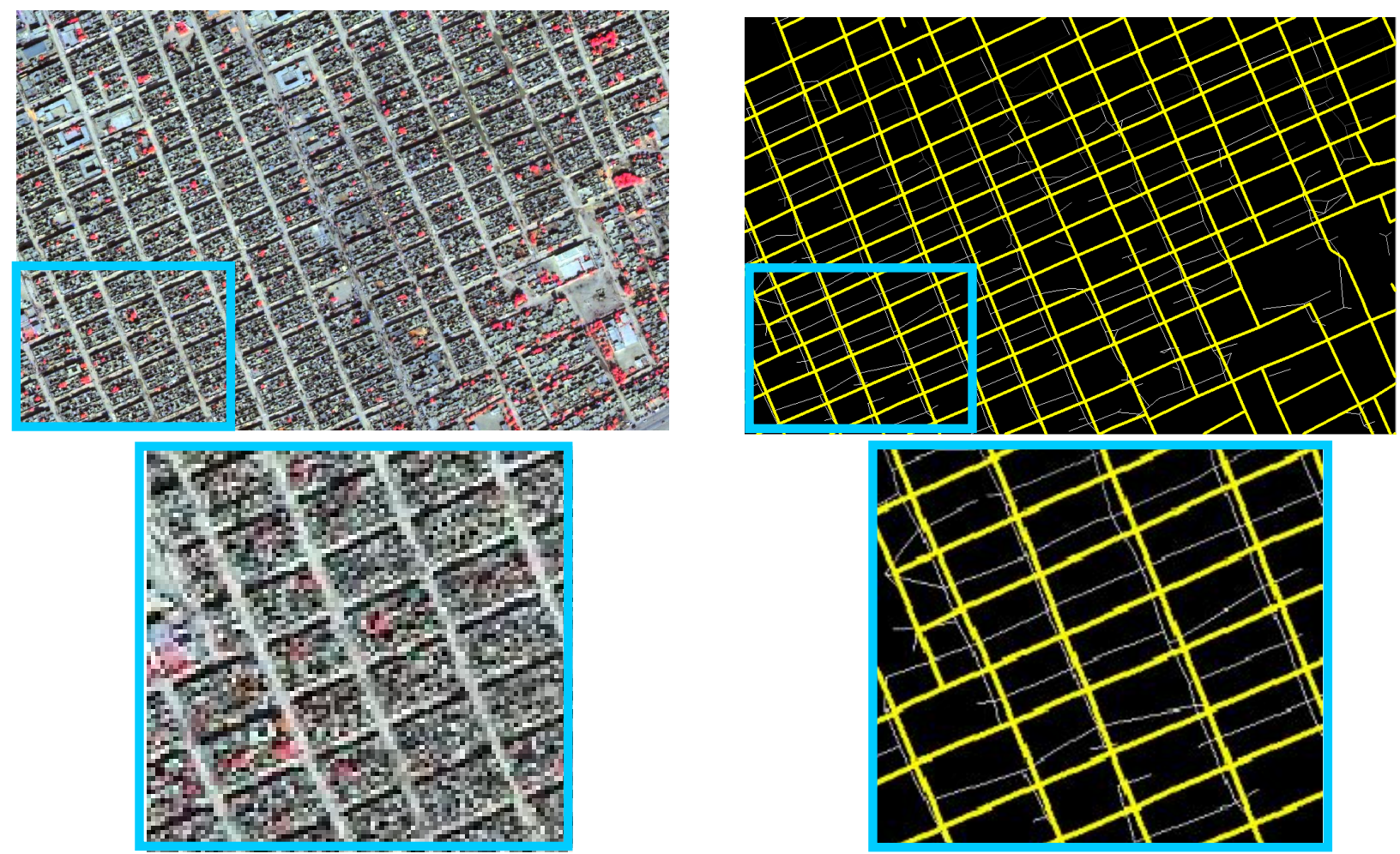

(a)

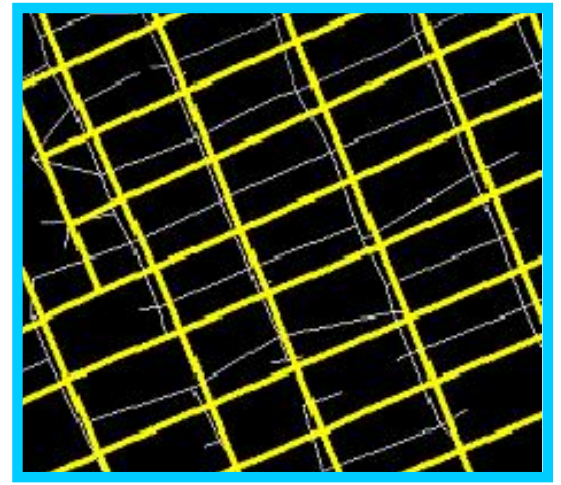

(b)

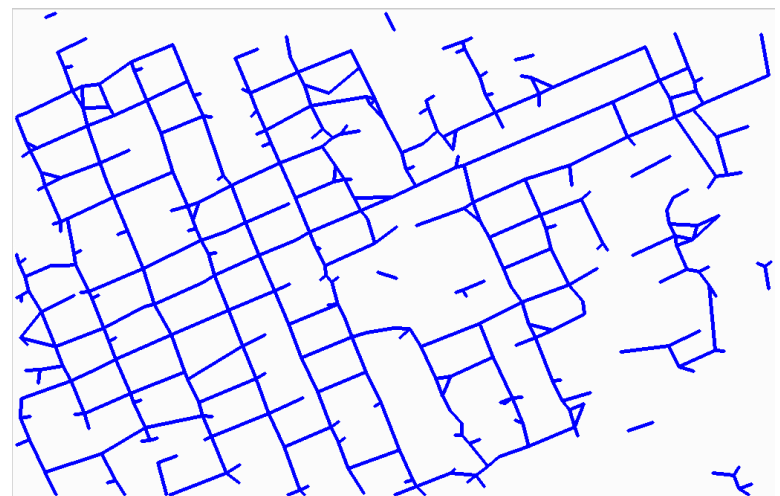

(c)

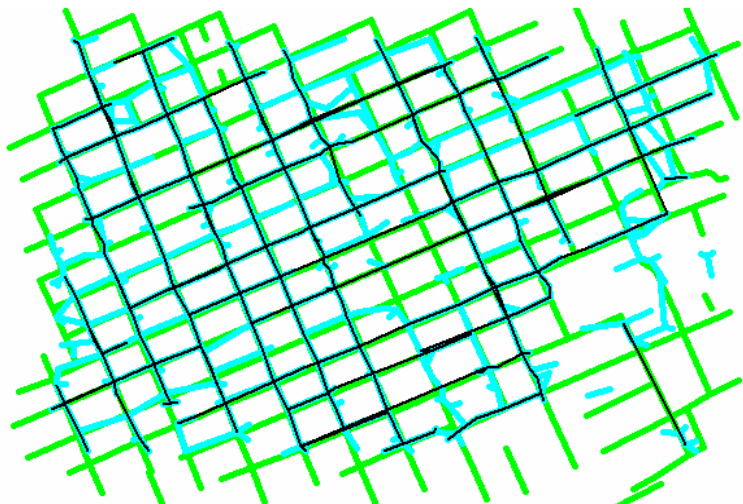

(d)

Fig. 6. Experiment with ASA method. (a) QuickBird MSI (CDigitalGlobe, 2006); (c) road extraction output from Feature Analyst ${ }^{\circledR}$; (b) original road vectors (yellow) superimposed with Feature Analyst output (white); (d) final adjustment for original vectors (green), superimposed with Feature Analyst vectors (cyan), and ASA matched segments (black). 


\subsection{Similarity Transformation of Local Features (STLF)}

STLF is an extension of the method developed in ${ }^{10}$ that is used to match road centerlines that were obtained from vector data. The locality of STLF stems from the fact of partitioning the GIS vector data as well as the imagery into small tiles. And the matching process is performed over these local tiles (Fig. 7). The tiling approach is chosen to mitigate the global non-linearity of the transformation between vector data and imagery. For example, the three overlapping rectangles (red, blue, and yellow) shown in Fig. 7 represent local tiles in the imagery and vector data that need to be aligned or conflated automatically using STLF. This tiling is repeated over the whole image and the vector data. In this paper, three experiments were reported to demonstrate some of the important features of STLF, which are very critical to the overall success of the automatic alignment between vector data and imagery. In particular, the following issues were addressed:

1. The impact of incomplete road centerline hypotheses or gaps on the robustness of the matching process.

2. The impact of the geometric distribution of the road centerlines in imagery and vector data on the correctness of the matching process.

3. The impact of outliers in the vector data and imagery on the correctness of the matching results. Outliers here refer to incorrect matches or non identical information in the vector data and imagery.

4. The impact of the size or tiling on the consistency of the matching results.

Three local image tiles or patches, of different sizes, were extracted from the left upper corner of the image and the vector data (Fig. 7c). The red patch resembles a local area of $100 \times 100$ pixels; the blue one $200 \times 200$ pixels, and the yellow one is $400 \times 400$ pixels. The sizes of these patches were determined empirically at this stage of the research and indeed a sophisticated criterion is needed. In Fig. 8, the magenta color in each patch refers to hypotheses of road centerlines that were extracted from each image patch. The blue one refers to the vector data before alignment.
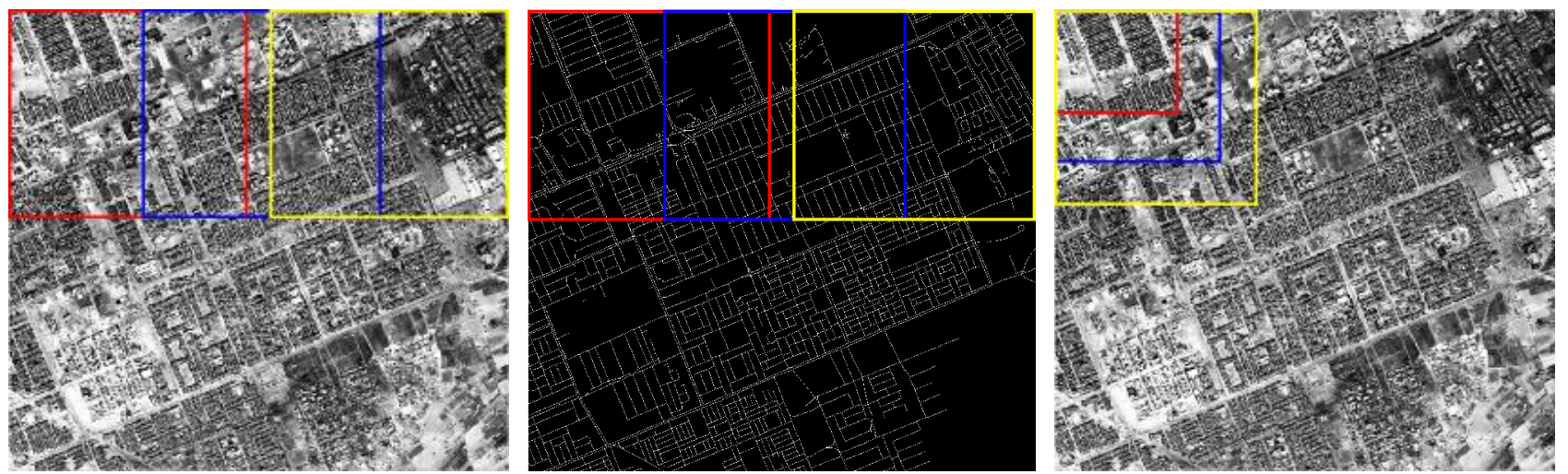

Fig. 7. Tiling in STLF. (a) across image; (b) on corresponding road vectors; (c) experiments with different tile sizes.

Fig. 8. Patches of road centerlines and GIS vector data.

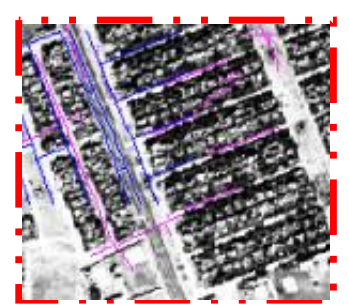

Red patch

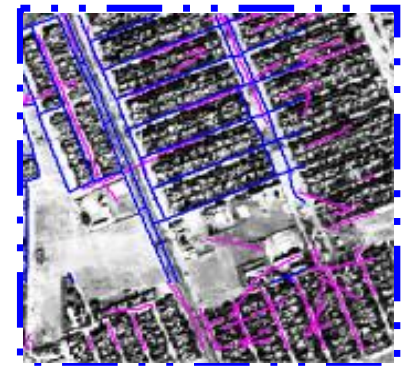

Blue patch

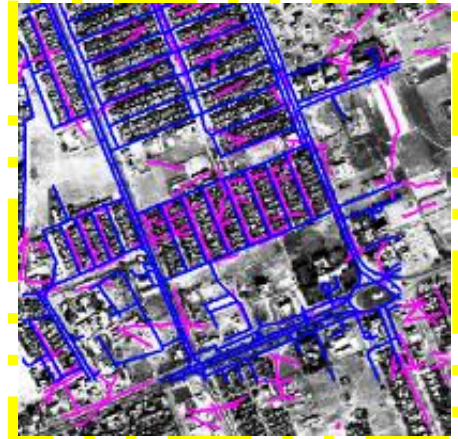

Yellow patch 
The STLF algorithm was used to match the vector data with the hypotheses of road centerlines. The parametric result of this matching is shown in Table 2. Few matching results were obtained from the red patch (the smallest one) and this is could be explained by the limited number of similar information between the vector data and imagery centerlines. An abundance of matching results was obtained from the blue and yellow patches, which show more consistent results in the transformation parameters. This is explained by the larger number of similar information between the vector data and imagery centerlines in the larger tiles. An informative and objective criterion needs to be developed to address the issue of the size selection of the local patch. The incomplete road centerlines or gaps that were inherited from the feature extraction algorithm (in this case, ACE), are not problematic for STLF to retain correct matching results.

Table 2. Similarity transformation parameter results from STLF for each patch (two translations, scale, and rotation).

\begin{tabular}{ll}
$X T$ (pixel) & 2.9649 \\
\hline$Y T$ (pixel) & 0.58088 \\
\hline Scale & 0.9965 \\
\hline Rotation Angle (degrees) & 0.087252 \\
\hline
\end{tabular}

Red patch.

\begin{tabular}{ll}
$X T$ (pixel) & 4.3042 \\
\hline$Y T($ pixel) & 0.42491 \\
\hline Scale & 1.0008 \\
\hline Rotation Angle (degrees) & -0.046057 \\
\hline
\end{tabular}

Blue patch.

\begin{tabular}{ll}
$X T$ (pixel) & 4.3157 \\
\hline$Y T$ (pixel) & 0.40744 \\
\hline Scale & 1.0003 \\
\hline Rotation Angle (degrees) & -0.00044838 \\
\hline
\end{tabular}

Yellow patch.

\section{CONCLUSIONS AND FUTURE WORK}

Results from AFE algorithms used for road extraction indicated better results from Feature Analyst versus ACE. This result is expected since the former is semi-automated (human supervised), and the latter fully automated. The multispectral imagery $(4 \mathrm{~m})$ provided better AFE road results than the panchromatic or pan sharpened imagery $(1 \mathrm{~m})$. This result is likely due to smoothing effects to the AFE output when using lower resolution imagery, which dramatically reduced false positives from scene clutter.

Current experiments have qualitatively shown that BSD-based ASA, and STLF are able to provide meaningful road matching with a partial solution to automated road extraction. Important functionalities demonstrated include: 1) matching polylines that have gaps; 2) measuring level of structural match and mismatch; and 3) abilities of measuring structural line discrepancy in meters.

We believe there is a clear tradeoff between (1) minimizing time for matching points (by matching fewer numbers of points) and (2) minimizing time for manual inspection to ensure that the match is sufficiently accurate. Any approach that minimizes item (1) will likely increase item (2) to maintain a comparable level of robustness over a wide range of images. This tradeoff issue can be addressed in an innovative way by designing an intelligent switching mechanism between automatic and interactive modes. This includes two complementary V-to-I methods that can be used to 1) perform cross verification, and 2) internally design algorithms that can simulate human inspection of results by employing a structural mathematical approach versus a more traditional distance-based approach.

Results from experiments presented in this paper were intended to be qualitatively evaluated by visual inspection in support of a proof-of-concept approach to vector-to-image registration. The next stage of development is expected to consider rigorous quantitative evaluation and validation. In this section, we describe the intended approach. Our approach will be to manually extract ground truth from the image as vectors, then rasterize the centerlines for a pixel-bypixel comparison with AFE output. This will allow for comparison of intermediate (shape points) between different road extractions. In order to accommodate variances in the extraction process, a buffer will be established around the centerline. To the extent that road extraction variances go beyond the buffered truth region, overly optimistic or pessimistic measures of correctness and completeness may result in an absolute sense. However, extraction variances should have less impact when making relative accuracy comparisons.

The completeness and correctness performance measures will be used with an adjustable buffer to assess between nodes segments, as well as operational sensitivity of the method that relates AFE accuracy to accuracy of registration. An assessment of operational conditions (e.g., image quality, scene content, imaging geometry, spatial resolution, road contrast and resolvability relative to background, etc.) will be conducted. Sensitivity analysis will determine how the relative performance of AFE roads propagates to, and impacts the performance of V-to-I registration. 


\section{ACKNOWLEDGEMENTS}

This work was funded by the US Army Topographic Engineering Center through contract number W9132V-07-C-0002.

The authors would like to thank Jamal Beck and Lou Martucci for their support.

\section{REFERENCES}

[1] Chen, C., C. Knoblock, C. Shahabi, C. Thakkar, and Y. Chiang, "Automatically and Accurately Conflating Orthoimagery and Street Maps", Proceedings of the 12th ACM International Symposium on Advances in Geographic Information Systems (ACM-GIS'04), 47 - 56 (2004).

[2] Cobb, M., M. Chung, M. Foley, F. Petry, K. Shaw, and H. Miller, "A Rule-based Approach for the Conflation of Attributed Vector Data", GeoInformatica, 2 (1), 7-36 (1998).

[3] Kovalerchuk, B, and J. Schwing, (eds.), [Visual and Spatial Analysis: Advances on Data Mining, Reasoning and Problem Solving], Springer, (2005).

[4] Brown, L., "A Survey of Image Registration Techniques", ACM Computing Surveys, 24(4), 325-376 (1992).

[5] Lewis, D., S. Bergeron, M. Kim, and P. Doucette, "Automated Registration Evaluation System (ARES)", AIPR Workshop, IEEE Comp. Soc., Wash D.C., (2007).

[6] Doucette, P., P. Agouris, and A. Stefanidis, "Automated Road Extraction from High Resolution Multispectral Imagery”, Photogrammetric Engineering \& Remote Sensing. 70(12), 1405-1416 (2004).

[7] Brigantic, R., P. Doucette, and R. Mendoza, "Sensitivity Analysis of Model Parameters for Automated Road Extraction from Remotely Sensed Images", Pacific Northwest National Laboratory, Report PNWD-3810, (2007).

[8] Kovalerchuk, B., Y. Kamatkova, and P. Doucette, "Overcoming the Combined Effect of Geometric Distortion and Object Change in Image Registration and Conflation", Proc. SPIE, v. 6233 - Algorithms and Technologies for Multispectral, Hyperspectral, and Ultraspectral Imagery, 62331S, (2006).

[9] Kovalerchuk, B., and R. Chase, "Image Analysis Method for Overcoming Source Distortion Using Algebraic Invariant Methods", SPIE Defense and Security Symposium, Orlando, FL, (2005).

[10] Seedahmed, G., and L. Martucci, "Automated Image Registration Using Geometrically Invariant Parameter Space Clustering (GIPSC)", Archives of the ISPRS, Commission III Symposium, September 9-13, Graz, Austria, (2002).

[11] Lavigne, D., Gang Hong, Yun Zhang, Performance Assessment of Automated Feature Extraction Tools on High Resolution Imagery, MAPPS/ASPRS 2006 Fall Conference, San Antonio, Texas, http://www.vlsinc.com/feature_analyst/publications/success/AFE-Ecog-FA.pdf, (2006)

[12] Wiedemann, C., Heipke, C., Mayer, H. \& Jamet, O., Empirical Evaluation of Automatically Extracted Road Axes. In: Empirical Evaluation Methods in Computer Vision (eds. Bowyer, K. \& Phillips, P.), IEEE Computer Society Press, 172-187 (1998).

[13] Box, G. and Draper, N., [Empirical Model-Building and Response Surfaces]. New York: John Wiley \& Sons, (1987).

[14] Montgomery, D., [Introduction to Statistical Quality Control] (Second Edition). New York: John Wiley \& Sons. (1991) 